\title{
Sensing Lower Body Lifting Posture through Disposable Sensing Coveralls
}

Cheol-Hong Min, Minnesota State University, Mankato, USA

Crystal Compton and Lucy E. Dunne,

University of Minnesota, USA

Keywords: Coveralls, e-textiles, wearable sensors, wearability, posture related injuries.

Introduction: Integration of sensors into clothing shows potential for many health and wellness applications, including the prevention of posture related injuries. Sensors that are seamlessly integrated into clothing eliminate the invasiveness and bulk that can be present with traditional sensor devices, providing improvements in the wearability of sensing systems.

Back injuries are the most common type of work injuries in the U.S. today (Bereau of Labor Statistics, 2013). These injuries can occur when excessive force is applied to the back during lifting with poor posture. This study focuses on the detection of lower body posture to assist workers in the prevention of posture related injuries by integrating stitched stretch sensors into a commonly worn type of personal protective equipment (PPE), disposable coveralls.

Sensing Garment: An industrial coverstitch machine was used to seamlessly integrate stitched stretch sensors into the fabric of coveralls, following the technique described by Gioberto et al. (Gioberto \& Dunne, 2012). This technique produces an inexpensive and reliable e-textile sensor, feasible for use in a disposable garment. Conductive thread (Shieldex 234/34) was used for the bottom cover thread in this machine, in order to construct and form the sensor. An array of 6 sensors, each 9 inches long, was stitched vertically along the limb axis on the dorsal hip and ventral knee of a commercial non-woven coverall to detect leg position during lifting, as seen in Fig. 1.

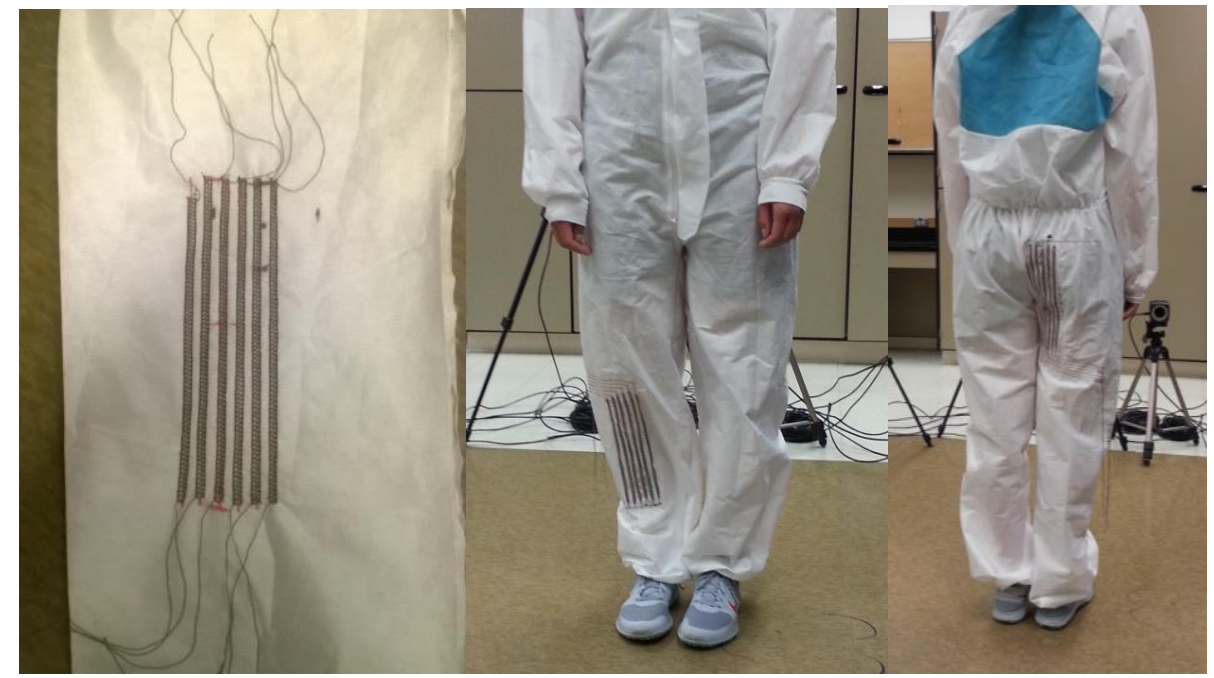

Figure 1. Sensor array (left), coverall front (middle) and coverall back (right)
Method: Data was collected from 6 different participants. For each knee and hip recording, each participant lifted a moderately heavy box while carrying out both good and bad postures, 10 times for each posture. The good posture required the participant to squat,

Page 1 of 2 
and the bad posture required the participant to bend in order to pick up the box. A digital multi-meter was used to record the resistance response of each sensor during the trial.

Due to the fact that coveralls are quite generic (in order to "fit" a wide variety of people) and loose fitting, sensors therefore fall in different locations on the body for different wearers. This creates a range of resistance readings for different participants. To simulate an adaptable system design, for each participant the sensor readings for the entire array of both the knee and hip joints were collected, and the sensor with the maximum range was used.

A Gaussian-mixtures model (GMM) machine-learning technique was used to classify the sensor data in order to identify postures. Activities not classified as "bend" or "squat" were grouped as "other activity" (OA). The classifier accuracy results are shown in Table 1.

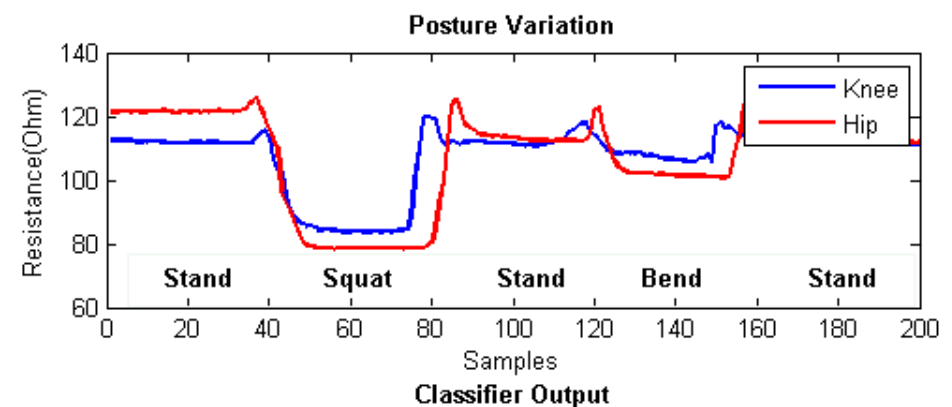
Results: Analysis of the data captured showed that there was overlap present between stand and bend postures. However, a good distinction of good lifting posture exists. However, since classification accuracy for "squat" (good posture) is higher than that of "bend" (bad posture), warning a wearer of bad posture may be more difficult than the rewarding of good posture.

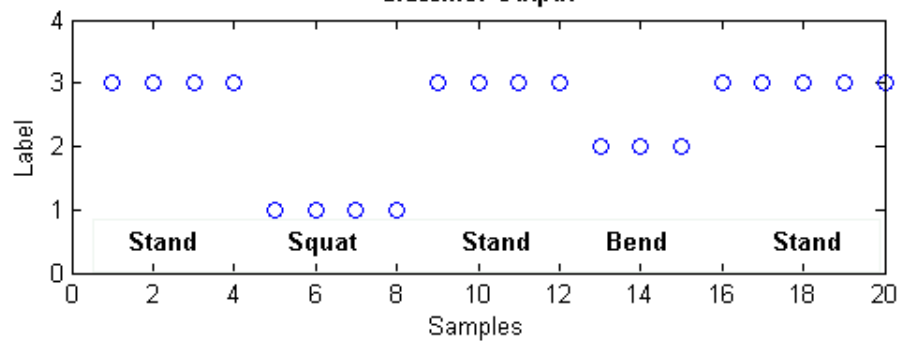

Acknowledgements: This work was supported by the National

\begin{tabular}{|c|c|c|c|c|}
\hline & OA & Stand & Squat & Bend \\
\hline Accuracy & $97 \%$ & $81 \%$ & $87 \%$ & $73 \%$ \\
\hline
\end{tabular}
Science Foundation under grant \#IIS-1116719, and by the Minesota Agricultural Experiment

Figure 2. Sensor output (top), classifier output (middle), and overall classifier accuracy (bottom) Station under grant MIN-53-042.

\section{References:}

Bereau of Labor Statistics. (2013, November 26). Nonfatal Occupational Injuries and Illnesses Requiring Days Away From Work, 2012. U.S. Department of Labor. Gioberto, G., \& Dunne, L. E. (2012). Coverstitched Textile Stretch Sensors: Fabrication and Characterization. In Proceedings of the IEEE Conference on Systems, Man, and Cybernetics. Seoul, Korea. 